Revue d'histoire de l'Amérique française

REVUE D.HISTOIRE DE L'AMÉRIQUE FRANÇAISE

\title{
Temps industriel et temps libre à Trois-Rivières : une étude de cas
}

\section{Gilles Pronovost et Pierre Girard}

Volume 41, numéro 2, automne 1987

URI : https://id.erudit.org/iderudit/304551ar

DOI : https://doi.org/10.7202/304551ar

Aller au sommaire du numéro

Éditeur(s)

Institut d'histoire de l'Amérique française

ISSN

0035-2357 (imprimé)

1492-1383 (numérique)

Découvrir la revue

Citer cet article

Pronovost, G. \& Girard, P. (1987). Temps industriel et temps libre à

Trois-Rivières : une étude de cas. Revue d'histoire de l'Amérique française, 41(2), 205-232. https://doi.org/10.7202/304551ar
Résumé de l'article

Le but de cet article est d'illustrer, à partir de l'exemple d'une entreprise trifluvienne de la chaussure, comment concrètement le temps de travail a été progressivement structuré, lors du processus d'industrialisation qu'a connu le Québec au siècle dernier. Or, ce n'est pas seulement le travail qui a été ainsi structuré, mais l'ensemble des temps sociaux. Dans le cas du temps libre notamment, dirigeants et ouvriers qualifiés de l'entreprise de chaussure étudiée ont aussi été à l'origine de la mise en place de nouvelles activités sociales, culturelles et sportives que décrit la suite de l'article. La structuration du temps libre est concomitante de la structuration du temps de travail; changements culturels et changements économiques vont de pair.
Tous droits réservés @ Institut d'histoire de l'Amérique française, 1987
Ce document est protégé par la loi sur le droit d'auteur. L'utilisation des services d'Érudit (y compris la reproduction) est assujettie à sa politique d'utilisation que vous pouvez consulter en ligne.

https://apropos.erudit.org/fr/usagers/politique-dutilisation/ 


\title{
TEMPS INDUSTRIEL ET TEMPS LIBRE A TROIS- RIVIERES: UNE ÉTUDE DE CAS ${ }^{1}$
}

\author{
GILLES PRONOVOST \\ PIERRE GIRARD \\ Département des sciences du loisir \\ Université du Québec à Trois-Rivières
}

\begin{abstract}
RÉSUMÉ
Le but de cet article est d'illustrer, à partir de l'exemple d'une entreprise trifluvienne de la chaussure, comment concrètement le temps de travail a été progressivement structuré, lors $\mathrm{du}$ processus d'industrialisation qu'a connu le Québec au siècle dernier. Or, ce n'est pas seulement le travail qui a été ainsi structuré, mais l'ensemble des temps sociaux. Dans le cas du temps libre notamment, dirigeants et ouvriers qualifiés de l'entreprise de chaussure étudiée ont aussi été à l'origine de la mise en place de nouvelles activités sociales, culturelles et sportives que décrit la suite de l'article. La structuration du temps libre est concomitante de la structuration du temps de travail; changements culturels et changements économiques vont de pair.
\end{abstract}

\section{ABSTRACT}

Based on a case study reviewing the development of a Trois-Rivières shoe factory, this article aims at illustrating the evolution of working time which took place within the more general industrialization process in the province of Québec during the last century. It shows that all social times underwent this process, including working time. The case of this particular shoe factory is an example of how managers and skilled workers contributed to the organization of new leisure time in the form of new social, cultural and sports activities, which are described in the rest of the article. Thus it seems that the structuration of working time, that economic changes go hand in hand with cultural changes.

\section{INTRODUCTION}

Cet article vise à illustrer, par une étude de cas, le processus de la formation simultanée du temps industriel et du temps libre au Québec au tournant du siècle dernier. Nous avons choisi de prendre en considération une industrie de la chaussure localisée dans une ville qui a connu des phases caractéristiques du mouvement d'industrialisation: Trois-Rivières.

1 Cette recherche a été réalisée grâce à l'appui financier du fonds FCAR (Québec) et du Conseil de recherche en sciences humaines du Canada. Nous tenons à remercier Messieurs René Hardy et Normand Séguin, du Centre de recherche en études québécoises, de l'UQTR, pour leurs commentaires judicieux. 
Par-delà des choix de convenance, notre propos était de pouvoir observer comment, en pratique, s'est effectué le "passage» vers un temps industriel de plus en plus structuré, et comment, de façon concomitante, le temps libre moderne à son tour a pris forme. Il était donc capital que l'étude de cas porte sur une «manufacture» typique de la fin du siècle dernier et que celle-ci soit localisée dans un milieu urbain ayant déjà une histoire assez longue pour qu'on puisse y observer les transformations induites par l'industrialisation (ce qui excluait les villes créées de toutes pièces par l'implantation de grandes entreprises).

Nous avons choisi de nous concentrer sur la structuration et la transformation des rapports entre deux catégories de temps sociaux:

1 - Le temps industriel, défini par la synchronisation progressive du travail en usine à partir de l'industrialisation, dont le corollaire est l'imposition d'une «discipline du temps de travail»; plus généralement, il s'agit de la transformation des rapports sociaux de production, dont des rapports à un temps de plus en plus spécifique faisant l'objet de calculls proprement économiques; un tel temps occupe progressivement une place centrale dans les sociétés de type «capitaliste» et devient le temps-pivot autour duquel l'ensemble des autres temps sociaux seront réaménagés. Ainsi, non seulement les conceptions sociales du temps de travail changent (le temps devient «marchandise», il acquiert une "valeur»), mais aussi les acteurs qui contrôlent les conditions d'organisation des temps sociaux.

2 - Le temps libre, lequel constitue l'une des catégories de temps résultant du ré-aménagement progressif de l'ensemble des temps hors travail en fonction de la centralité croissante du temps industriel. Historiquement, le temps libre a été conçu comme un temps «gagné» sur le travail, souvent indistinctement associé à l'origine au temps scolaire, au repos, à la récupération physique, au «divertissement», mais acquérant progressivement des finalités et des contenus qui lui sont propres et d'où est issu, en partie, le loisir moderne.

Sans présumer qu'il s'agisse des seuls temps sociaux significatifs, nous avons choisi de limiter le projet à ceux-ci, pour des raisons à la fois théoriques - dont la centralité croissante du temps industriel et l'étude du processus de formation du loisir moderne, - et pratiques restreindre l'envergure du projet.

Après avoir présenté le contexte de l'implantation de l'usine de chaussures que nous avons choisi d'observer, nous analyserons plus en détail les modalités effectives selon lesquelles y a été structuré le temps industriel et, par la suite, nous décrirons la structuration concomitante de quelques-unes des principales activités sociales, culturelles et sportives qui ont eu pour acteurs principaux les dirigeants et les ouvriers qualifiés de cette même manufacture. 


\section{1 - Trois-Rivières: industrialisation et transformation des structures sociales}

Brosser un tableau des transformations lentes mais profondes que subit Trois-Rivières dans le dernier tiers du siècle dernier ${ }^{2}$, déborde largement le cadre de cet article. Pour les fins de notre propos, nous soulignerons surtout que l'organisation du travail y est en pleine mutation, ce que l'on peut constater notamment par les transformations observables dans la structure des professions et par l'arrivée sur la scène économique d'un nouveau type de dirigeant: l'immigrant audacieux qui a intériorisé les règles du jeu capitaliste de l'époque.

Au milieu du siècle dernier, Trois-Rivières, avec ses 5000 habitants, constituait la $3 \mathrm{e}$ ville en importance au Québec, suivie de près par Sherbrooke. Sur la rive nord du Saint-Laurent, elle représentait le seul véritable centre urbain entre Montréal et Québec; marquée, comme on le sait, par l'industrie du bois, «le sciage et ses activités connexes et dérivées (qui) comptaient pour plus de la moitié des emplois non identifiés au commerce et aux services durant toute la seconde moitié du $19 \mathrm{e}$ siècle» ${ }^{3}$. Mais il ne s'agissait pas du seul type existant d'entreprises; l'implantation d'entreprises, dans le secteur du cuir et des métaux tout particulièrement, illustre «que la ville parvenait à la fin du siècle à s'appuyer sur d'autres bases que l'exploitation de la forêt et le sciage pour assurer sa croissance» ${ }^{4} ; l^{\prime}$ entreprise de chaussures que nous avons choisi d'étudier en constitue une claire illustration.

Ajoutons encore que Trois-Rivières constituait nettement une ville administrative et de services; on y retrouve une administration judiciaire, des journaux locaux, et l'ouverture de plusieurs succursales en font un centre de services bancaires non négligeable. La ville n'était pas isolée puisque le port se développe de façon importante dans la seconde moitié du 19e siècle, qu'un chemin de fer entre en opération dès 1879 et qu'un réseau routier prend forme.

\section{A - La structure des professions}

En consultant les données agrégées des recensements, on peut y constater un exemple de croissance industrielle caractéristique des économies en transformation. En premier lieu, paradoxalement, le nombre d'industries diminue: entre 1871 et 1901, le nombre d'industries recensées se réduit de $400 \%$, sans doute sous l'effet de la disparition rapide d'un grand nombre de fabriques artisanales ou d'entreprises de petite

\footnotetext{
2 Pour références, on peut tout particulièrement consulter: Alain Gamelin, René Hardy, Jean Roy, Normand Séguin et Guy Toupin, Trois-Rivières illustrée (Trois-Rivières, la Corporation des fêtes du 350e anniversaire, 1984), 228 p. Annexes.

3 Alain Gamelin et al., op. cit. 35.

4 Ibid.
} 
taille (le nombre d'entreprises recensées passe en effet de 126, en 1871, à 31 , en 1901) $)^{5}$.

En second lieu, la taille des entreprises s'accroît considérablement puisque le nombre moyen d'employés par industrie est multiplié par 4. Nos décomptes personnels indiquent en effet une moyenne de 8,2 employés par entreprise, en 1871, par rapport à une moyenne de 38,3 en 1901. On note encore un pourcentage de main-d'oeuvre féminine oscillant entre le tiers et le quart de la main-d'oeuvre totale, et un pourcentage de main-d'oeuvre enfantine oscillant autour de 5\%.

Quant à la structure des professions, elle indique à son tour une mutation lente mais sans équivoque (tableau 1). Ainsi, le poids relatif des «servantes et domestiques» qui avait contribué à gonfler le ratio de population féminine diminue progressivement; de même en est-il du poids démographique des professions libérales qu'une ville traditionnelle de services tend à maintenir. Les artisans et les apprentis voient également leur importance relative diminuer. D'autre part, on peut noter un accroissement lent mais régulier de la main-d'oeuvre des secteurs publics et des services. Mais le phénomène le plus remarquable est la constitution de la classe ouvrière, gonflée de travailleurs des nouvelles manufactures, et qui va occuper près de $40 \%$ de l'ensemble des emplois: «journaliers», «ouvriers d'usine», etc.

Même si l'industrialisation de Trois-Rivières ne prend une certaine ampleur qu'au tournant du siècle, l'étude de la structure des professions révèle que des mutations typiques ont déjà commencé à se manifester bien avant; à ce chapitre, Trois-Rivières est une ville en phase d'industrialisation dès le début de la deuxième moitié du $19 \mathrm{e}$ siècle et de nouvelles structures sociales se mettent en place; lorsque l'essor industriel se manifestera, au 20e siècle, la ville sera déjà une ville industrielle au plan des professions.

\section{B - Un nouvel entrepreneurship: l'exemple d'une industrie de la chaussure}

L'implantation d'une industrie de la chaussure à Trois-Rivières s'inscrit dans les démarches courantes à l'époque, de la part des dirigeants municipaux, en vue d'attirer les investissements.

Les journaux trifluviens font mention de quelques tentatives en ce sens, en 1883. Mais il faudra attendre encore cinq ans avant qu'un manufacturier de chaussures de Montréal procède à des ententes for-

\footnotetext{
5 Rappelons qu'il s'agit de décomptes au sujet desquels de nombreuses nuances devraient être apportées; les données sont tirées des recensements agrégés; elles sont reproduites, avec une critique des sources, dans Pierre Lanthier et Alain Gamelin, L'industrialisation de la Mauricie, dossier statistique et chronologique 1870-1875 (Trois-Rivières, UQTR, Groupe de recherche sur la Mauricie, 1981), 34.
} 
melles (construction d'un bâtiment, engagement de 200 employés), en contrepartie d'une subvention de $35000 \$$ et d'exemptions de taxes pour dix ans ${ }^{6}$.

Les modalités de financement utilisées par cet entrepreneur sont sans doute typiques de l'époque. La petite société créée est en grande partie financée par des banques montréalaises; l'investissement, éventuellement récupéré par la subvention municipale, ne porte que sur la construction de l'édifice puisque la machinerie est louée à des compagnies américaines; le Conseil municipal ayant imposé le déménagement de ses affaires à Trois-Rivières, l'entrepreneur occupe un terrain loué

TABLEAU 1

Structure des professions, Trois-Rivières*, 1861-1881

\begin{tabular}{|lrr|rr|rr|}
\hline PROFESSIONS & \multicolumn{2}{|c|}{1861} & \multicolumn{2}{|c|}{1871} & \multicolumn{2}{|c|}{1881} \\
& $\mathrm{~N}$ & \multicolumn{1}{c|}{$\%$} & $\mathrm{~N}$ & $\%$ & \multicolumn{1}{c|}{$\mathrm{N}$} & $\%$ \\
\hline Professions libérales, rentiers, & 148 & 9,5 & 178 & 7,2 & 133 & 4,9 \\
propriétaires fonciers & & & & & & \\
Employés de bureau, officiers, commis & 131 & 8,4 & 202 & 8,2 & 265 & 9,8 \\
Marchands, commerçants, gérants & 137 & 8,8 & 209 & 8,4 & 244 & 9,0 \\
Artisans et apprentis & 148 & 9,5 & 178 & 7,2 & 133 & 4,9 \\
Ouvriers non spécialisés & 482 & 30,8 & 897 & 36,4 & 1031 & 38,1 \\
Ouvriers spécialisés & 148 & 9,5 & 283 & 11,5 & 354 & 13,1 \\
Agriculteurs et paysans & 80 & 5,1 & 177 & 7,2 & 126 & 7,2 \\
Servantes et domestiques & 271 & 17,3 & 244 & 9,9 & 170 & 6,3 \\
Religieux, ministres du culte & 18 & 1,2 & 90 & 3,7 & 118 & 4,4 \\
Divers, non classés & 1 & 0,1 & 6 & 0,2 & 63 & 2,3 \\
TOTAL & 1564 & & 2464 & & 2707 & \\
\hline
\end{tabular}

6 Le Journal des Trois-Rivières, 1er, 2 et 12 décembre 1887, 2.

* District de recensement de Trois-Rivières.

Source: décomptes personnels des recensements. Ces décomptes personnels relèvent de notre propre interprétation des catégories nominales utilisées par les recensements; ils ont uniquement pour objet de dresser un portrait approximatif de l'évolution de la structure des emplois et des professions. Voici une liste partielle indicative des rubriques du tableau 1: «professions libérales...»: architectes, avocats, banquiers, juges, notaires, rentiers, etc.; «employés de bureau...»: commis, employé du gouvernement, gardien, instituteurs, etc.; «marchands...»: boulanger, boucher, hôtelier, marchand, négociant, etc.; «artisans...»: apprentis, batelier, carossier, charretier, sellier, faiseur de roue, tonnelier, etc.; «ouvriers non spécialisés»: journalier, ouvrier d'usine, etc.; «ouvriers spécialisés...»: charpentiers, ferblantier, fondeur, forgeron, mécanicien, plombier, etc.; les autres rubriques sont une reproduction quasi textuelle des catégories des recensements. 
par la ville et engage une partie de la main-d'oeuvre qu'il a mise en chômage à Montréal. Bref peu ou rien ne lui appartient. On dira qu'il se contente de «faire circuler le capital» en tentant d'y retenir une petite partie des bénéfices.

D'où la très grande vulnérabilité de la manufacture aux moindres aléas de la conjoncture économique, au point de contraindre son propriétaire à faire cession de ses biens au profit de ses créanciers par deux fois, soit en avril 1893 et en février 1894. Finalement, Richard Smardon doit déposer son bilan, au début de l'année $1896^{7}$.

L'entreprise reste inactive pendant le reste de l'année 1896, puis est rachetée, en mars 1897, par trois entrepreneurs en chaussures de la ville de Québec: Jean-Louis Thibodeau, Louis Beaubien et Georges Binet. Les affaires tournent au ralenti, la production s'arrête à quelques reprises, la ville doit consentir de nouvelles concessions; finalement, un des associés se retire, puis la manufacture ferme en mars 1900. La ville reprend la manufacture ${ }^{8}$.

\section{De l'apprenti au patron: John-Thomas Tebbutt}

Les frères Tebbutt récupèrent, le 17 avril 1900, la manufacture de la corporation municipale pour la somme de $2000 \$$, un investissement sans commune mesure avec la valeur réelle de la manufacture; autrement dit, ils achètent à bas prix une entreprise en faillite, pour tenter de réussir en affaires là où leurs prédécesseurs avaient échoué. La carrière de John-Thomas Tebbutt présente bien les traits du «self-made man» qui gravit les échelons d'une entreprise pour monter sa propre affaire après plusieurs années d'efforts acharnés. Il est l'incarnation même de l'entrepreneurship de l'époque. Né le 10 septembre 1860 dans la petite ville industrielle de Kettering, Northamptonshire, dans le nord industriel de l'Angleterre, il fait son apprentissage d'ouvrier de la chaussure dans une des nombreuses fabriques de la région. A l'âge de 24 ans, soit en 1884, il part avec sa famille pour aller s'installer à Montréal où il débute comme contremaître pour la Ames Holden Co. Il passe ensuite au service de R. Smardon comme surintendant de la manufacture de chaussures du même nom. Lorsque Richard Smardon déménage son entreprise à Trois-Rivières en 1888, John-Thomas Tebbutt est du nombre des employés qui partent de Montréal pour suivre leur patron.

John-Thomas, en plus de son travail de surintendant, parcourt le continent comme commis-voyageur pour le compte de Smardon. A cette époque, il «part avec ses valises d'échantillons pour l'Ouest cana-

\footnotetext{
7 Les renseignements qui précèdent sont tirés des Archives nationales du Québec, à TroisRivières, Cour supérieure, Cause 222, Liste des exhibits de la contestante à l'enquête Rochette, Smardon et Corporation de Trois-Rivières et Dessureault, boîtes 258, 259, 260, mai-juin 1896.

8 ANQ-TR, Cour supérieure, Cause 103, boîte 277, mars 1900.
} 
dien». Ces contacts avec le marché nord-américain le serviront lorsqu'il reprendra la manufacture en 1900 avec son frère James. Ses nombreux voyages lui permettront, dans les années 1910-1920, d'atteindre la clientèle du «Canada, du Cap-Breton à Vancouver et aux États-Unis, du Maine à Chicago» 9 .

Quelque temps après la faillite de R. Smardon en 1895, JohnThomas Tebbutt et son frère James-S. ouvrent un commerce de vente au détail de chaussures «Slater» sous la raison sociale Tebbutt Brothers. Tout en s'adonnant au commerce, John Tebbutt demeure contremaître pour la Three Rivers Shoe qui prend la relève de Smardon de 1896 à 1900. Les deux frères avaient aussi un entrepôt sur la rue Saint-Roch qui, semble-t-il, abritait un atelier de fabrication de chaussures. Tout au plus, sans doute, s'y occupait-on à remplir des sous-contrats pour la Three Rivers Shoe.

Dès 1896, les frères Tebbutt avaient fait les démarches nécessaires pour racheter la manufacture lors de la faillite Smardon en 1896. Ils s'engageaient alors à faire travailler 75 personnes pendant 300 jours par année. Mais on sait que leur offre fut rejetée au profit de Thibodeau, Beaubien et Binet. Lorsque les Tebbutt fondent leur compagnie en 1900, ils connaissent bien le marché et sont familiers avec les méthodes de production; ils ont acquis une «expérience» que n'avaient sans doute pas leurs prédécesseurs. Par exemple, au mois d'octobre de la même année, l'entreprise doit faire face aux revendications des cordonniersmonteurs de la manufacture qui exigent le respect des revendications salariales faites par le syndicat provincial et acceptées par plusieurs patrons des autres villes du Québec ${ }^{10}$. Les nouveaux patrons tiennent tête et la grève se termine par le renvoi des grévistes qui refusent de retourner au travail ${ }^{11}$.

La compagnie change de raison sociale en 1905 et passe de Tebbutt Brothers à The Tebbutt Shoe and Leather Company Limited (voir illustration). Les deux frères occupent alors la direction de l'entreprise. Er. fait, pour les Tebbutt, les affaires et la famille font un, si l'on se fie à l'image qu'ils projettent. Des débuts jusqu'en 1966, où la compagnie devient la propriété de Greb, l'entreprise est gérée par des membres de la famille qui en sont aussi les propriétaires ${ }^{12}$.

\footnotetext{
9 Renseignements tirés principalement du Who's Who (1914): 902 et de l'entrevue avec Jack Tebbutt, Le Nouvelliste, 22 août 1925, 13.

10 La Presse, 19 octobre 1900, 5.

11 Jean Hamelin, Paul Larocque et Jacques Rouillard, Répertoire des grèves dans la province de Québec au XIXe siècle (Montréal, 1970), 167.

${ }_{12}$ A noter que John-Thomas Tebbutt sera également impliqué dans la politique municipale: il est conseiller municipal de 1903 à 1907, et, à ce titre, semble s'être particulièrement intéressé aux questions d'hygiène et de santé.
} 


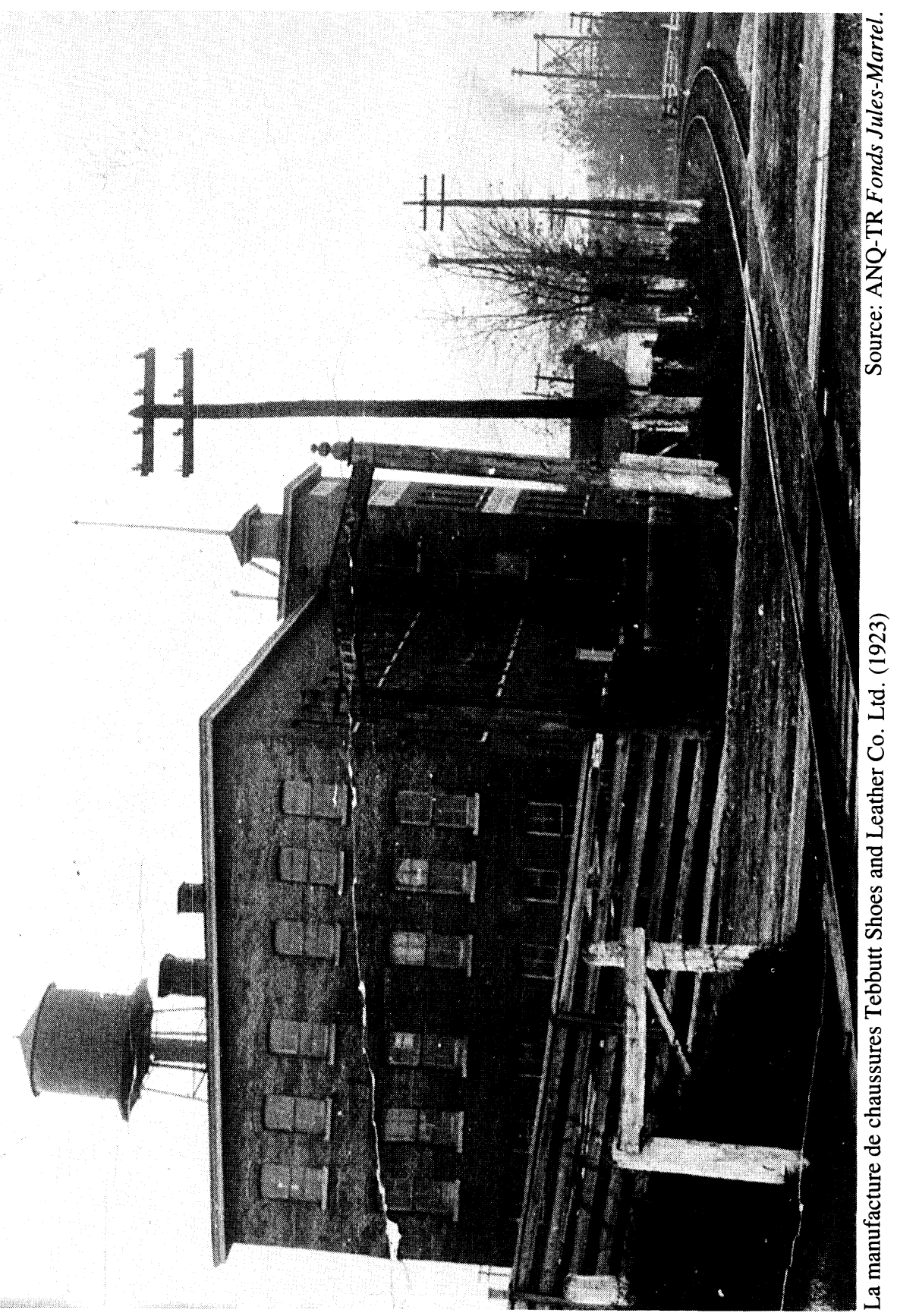


En résumé, les structures sociales de la ville sont en pleine mutation, principalement par le gonflement du nombre et de l'importance relative des ouvriers non spécialisés. Par ailleurs, la taille des entreprises s'accroît de façon significative, ce qui ne peut manquer d'apporter des changements majeurs dans l'organisation même des rapports de travail. L'exemple des frères Tebbutt montre aussi qu'un nouvel entrepreneurship prend forme, caractérisé notamment par l'audace, l'ouverture au marché nord-américain, l'acquisition de ce que l'on pourrait qualifier une «culture économique».

C'est sur cette toile de fond que sera maintenant illustré, par l'exemple de cette manufacture de chaussures, comment au moment de l'industrialisation de la ville de Trois-Rivières a pris forme et s'est développé le processus de structuration concomitant du temps de travail et du temps libre.

\section{2 - La formation du temps industriel}

Dans un texte désormais classique, Edward Thompson distingue deux grands types de mesure du travail ${ }^{13}$ :

1 - LDans un premier cas, il s'agit d'une mesure du travail «orientée par la tâche»; le travail ne fait pas l'objet de calculs précis, mais il est fonction du rythme des saisons, des périodes de chasse, de pêche, de semailles et de récoltes. Pour une analyse exemplaire de ce type d'organisation du temps, on peut se référer aux travaux célèbres d'Evans-Pritchard sur les Nuers ${ }^{14}$.

2 - À cause du processus de restructuration des temps sociaux qui s'opère dans les sociétés industrielles occidentales, la définition du temps y est tout à fait différente. C'est pourquoi Thompson parle cette fois de «tâche de travail mesurée par le temps», c'est-à-dire que l'on en arrive progressivement à effectuer des mesures de plus en plus précises pour calculer le temps consacré au travail. «Ce qui est déterminant», écrit Thompson, «ce n'est pas la tâche, mais la valeur du temps, une fois celui-ci converti en argent. Le temps est devenu monnaie» ${ }^{15}$.

On a ainsi assisté à un mouvement progressif, mais lent et irrégulier, de synchronisation du travail en usine. Il ne faut pas croire cependant que le mouvement s'est effectué spontanément, sans heurts et sans

\footnotetext{
13 E. P. Thompson, «Time, Work-Discipline and Industrial Capitalism», Past and Present, 38 (Dec. 1967): 56-97; traduction française, «Temps, travail et capitalisme industriel», Libre (no 5) (Paris, Payot, 1979).

${ }_{14}$ E. E. Evans-Prichard, Les Nuers (Paris, Gallimard, 1968). Nous avons développé ce thème dans notre article: «Introduction: une perspective historique et sociologique du temps», Revue internationale des sciences sociales, 107 (1987): 5-19.

15 Thompson, loc. cit., 10 de la traduction française.
} 
difficultés. A la lumière des données que nous avons recueillies et en y ajoutant des informations complémentaires (tirées notamment de la Commission d'Enquête de 1888 sur les rapports entre le capital et le travail), nous avons pu établir que le processus de structuration du travail industriel impliquait l'introduction de trois aspects majeurs: la régularisation du travail, la division du travail et la discipline de temps de travail. Encore une fois, l'industrie de la chaussure constituera notre référence typique.

\section{A - Du travail irrégulier au travail structuré}

L'irrégularité du travail se manifeste tant chez les ouvriers que chez les patrons. Pour les entrepreneurs, les difficultés d'approvisionnement, les problèmes d'énergie et de chauffage, les conjonctures économiques très variables, la mobilité de la main-d'oeuvre, donnent l'impression qu'il n'a pas été aisé de réussir à coordonner de manière harmonieuse tous les éléments nécessaires à une production régulière. Même en période de production, du fait que le travail est très parcellisé sans être entièrement synchronisé, des ouvriers qui sont affectés à une tâche particulière peuvent travailler de longues périodes de temps alors que les autres employés de la manufacture attendent que le matériel en préparation leur parvienne pour commencer à travailler ${ }^{16}$.

Les ouvriers subissent les contrecoups des aléas de la production, travaillant sans relâche en période de pointe, pour se retrouver en chômage quelques jours plus tard. La semaine et la journée pouvaient être tout autant irrégulières. On pouvait travailler tous les soirs, ou le samedi, selon les situations. Les heures de début et de fin du travail ont varié; même pendant les heures de travail, des témoignages illustrent qu'on quittait parfois l'usine soit tout simplement pour aller se chauffer chezsoi quand il faisait trop froid à la manufacture, ou bien plus souvent quand le manque de travail donnait l'occasion de prendre quelques heures de congé. Il semble aussi que les ouvriers ne commencent pas toujours à travailler à l'heure fixée par le patron surtout si le mauvais temps, l'hiver, gêne les déplacements ${ }^{17}$.

Enfin, c'est l'année entière qui était souvent tributaire de cycles de travail et de chômage. Dans le cas de la Smardon, il a été possible de situer le cycle annuel de travail (voir figure 1); les mois de mai et de juin amènent des phases de petites dépressions, tandis que novembre et décembre sont vraiment les deux mois creux du temps de travail. Ce cycle varie également d'un emploi à l'autre à l'intérieur d'une même manufacture: «... les tailleurs en général perdent au moins deux mois par année... tandis qu'un finisseur peut perdre, à peu près, toujours bien 2 à 3 mois dans l'année.» ${ }^{18}$

\footnotetext{
16 Commission royale d'enquête sur la relation entre le capital et le travail, 1888. Réponse d'un traiteur de cuir de Montréal (p. 272, 273, 412).

17 Ibid., 1093 et 1111 .

18 Ibid.
} 


\section{FIGURE 1}

Cycle de travail des employés de la manufacture de chaussures Smardon de Trois-Rivières (1889-1895).

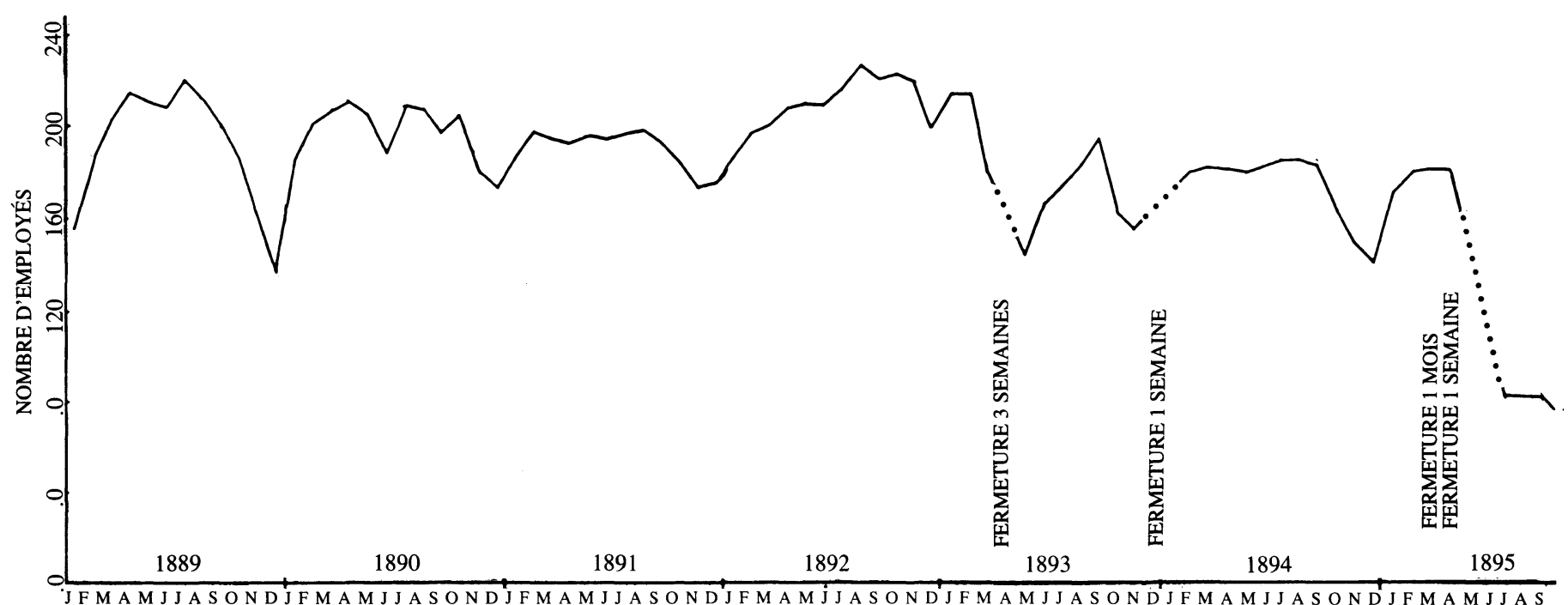

MOIS

Sources: ANQ-TR Archives judiciaires. Cour supérieure. Boîte 259. Cause 222. Liste des exhibits de la contestante à l'enquête du 16 mai 1896 dans l'affaire Rochette, Smardon, Corporation de Trois-Rivières et Dessureault. Exhibit no. 1 de la contestante de l'enquête. Rapport du secrétaire-trésorier de la corporation municipale de la ville de Trois-Rivières. 
Ainsi, les arrêts de production ne touchent pas les travailleurs au même instant et de la même manière. Les patrons revoient au jour le jour les conditions d'engagement. Cela leur permet de changer de poste un ou des employés, selon les besoins du moment. Lorsque la période de chômage approche, comme au mois de novembre, les employés font du temps supplémentaire. Par exemple, à la Three Rivers Shoe, le journal local rapporte, dans la semaine du 18 novembre 1898, qu'on «y travaille le soir». Une telle pratique se perpétue chez les Tebbutt, comme le précise le même journal en 1905: «Depuis quelques semaines, la manufacture Tebbutt Brothers marche le soir afin de pouvoir satisfaire aux nombreuses commandes qu'elle reçoit constamment. C'est une belle note pour MM. Tebbutt et pour nos industries trifluviennes.» ${ }^{19}$

Le témoignage du dernier dirigeant de l'usine est explicite quant au caractère irrégulier de cycle de la production jusqu'à l'époque récente: «La vente de chaussures était pas mal saisonnière et puis il y avait des périodes où la demande n'était pas bien forte. Alors des fois on travaillait seulement des semaines de 2, 3 et 4 jours. Des fois, on fermait pendant une semaine. ${ }^{20}$

Il n'est pas facile de distinguer clairement entre ce qui relève des fluctuations de la demande et ce qui est tributaire de l'organisation même du travail. Néanmoins, à l'instar de ce qui a été observé aux États-Unis, en France et en Angleterre, de telles situations témoignent que ce n'est que progressivement que le temps de travail a été régularisé, au plan des conditions de production, de l'ajustement aux conditions du marché, tout autant qu'au plan des «mentalités». Car en référence aux modèles de temps observables dans les sociétés pré-industrielles, il n'allait pas de soi que l'on se représente comme normale une conception du travail mesuré par le temps qu'on y consacre. La division du temps en périodes synchronisées par rapport au rythme du travail industriel n'a pu s'implanter que progressivement.

\section{$B$ - La division du travail}

Dans l'industrie de la chaussure, les années 1880 sont marquées par ce qu'on pourrait appeler la généralisation de l'usage des machines pour accomplir les tâches de production en manufacture. Des compagnies comme la Goodyear vont mettre au point des machines-outils capables de rivaliser avec l'habileté des artisans. Les compagnies offrent également les services de conseillers-techniques pour enseigner le fonctionnement du nouvel équipement aux employés qui devront l'utiliser. On retrouve de telles machines en location dans la manufacture trifluvienne.

19 Le Trifluvien, 3 octobre 1905, 8.

20 Entrevue avec Jack Tebbutt, dernier dirigeant survivant, loc. cit. 
Toutes les étapes de fabrication se subdivisent en plusieurs manipulations faites à l'intérieur et à l'extérieur de la manufacture. De 1880 à 1900 , le processus de production comprend environ 33 étapes. Entre 1900 et 1920 , on porte à plus de 100 le nombre de manipulations, faites de plus en plus avec l'aide des machines ${ }^{21}$. La Tebbutt Shoes ne fait pas exception à cette règle et, en 1925, la «fabrication d'une seule paire de chaussures requiert de 135 à 160 opérations».

La division du travail s'accompagne de la généralisation de l'embauche des femmes et des enfants pour s'occuper des machines venues remplacer les artisans. Les enfants sont surtout utilisés pour le transport des pièces et du matériel entre les différentes étapes de la fabrication et pour assister les adultes.

La mécanisation des entreprises s'est réalisée d'une manière progressive; dans une même manufacture, on peut retrouver plusieurs processus de fabrication qui en théorie se succèdent, mais qui en pratique coexistent.

Dans ce contexte, la manufacture de chaussures de Trois-Rivières se situe dans une phase intermédiaire entre le travail artisanal et le travail parcellisé. Ce n'est possiblement qu'en 1905 que les frères Tebbutt vont faire accéder la manufacture à une seconde phase de la division du travail. Cette année-là, ils réorganisent la production en installant l'électricité qui permet l'usage de nouvelles machines.

Une autre caractéristique de ce passage du mode artisanal à la manufacture est l'embauche d'employés non-spécialisés; ainsi Richard Smardon engage des Trifluviens sans expérience dans le travail de la chaussure. L'embauche de nouveaux employés s'appuie aussi sur les liens de parenté. Une certaine tradition de compagnonnage subsiste au sein de la structure familiale. Ce compagnonnage informel se perpétue même après les années 1920 dans les industries de la chaussure. Divers récits de vie, recueillis par des chercheurs de Québec, dont Nicole Thivierge, confirment que les travailleurs de la chaussure apprenaient le métier de leurs parents ${ }^{22}$. Le témoignage de Jack Tebbutt va dans le même sens:

C'était des fils, s'il y en avait, ça pouvait être d'autres personnes de la famille ou des amis. Anciennement surtout, c'était à ces garslà, les vieux employés, à choisir leurs apprentis. Comme pour

21 Jean-Claude Dupont et Jacques Mathieu, Les métiers du cuir (Québec, Presses de l'Université Laval, 1981); Blanche Evans Hazard, The Organization of Boot and Shoe Industry in Massachussetts before 1875 (Cambridge, Harvard University Press, 1921); John T. Cumbler, Working Class Community in Industrial America, Work, Leisure and Struggle in Two Industrial Cities (1888-1930) (Westport, Greenwood Press, 1939); Edgard Hoover, Location Theory and the Shoe and Leather Industries (Cambridge, Harvard University Press, 1937). Témoignage de Jack Tebbutt.

${ }_{22}$ Jean-Claude Dupont et Jacques Mathieu, op. cit., 371-413. 
n'importe quelle industrie si vous aviez un employé capable qui est en air de travailler, qui est un bon employé en tous les sens, et qu'il dit qu'il a un fils ou un neveu qui veut travailler, on aime mieux quelqu'un comme cela qu'un inconnu. ${ }^{23}$

Le «sweating-system» demeure une autre caractéristique du travail dans l'industrie de la chaussure. Les témoignages des travailleurs devant la CRERCT, en 1886, confirment que cette pratique existe autant à l'intérieur qu'à l'extérieur de la manufacture. C'est l'occasion pour le patron d'augmenter sa marge de profit en permettant d'exploiter une force de travail, surtout féminine, au-delà de ce qui se pratique avec les employés réguliers. Ce fonctionnement a subsisté jusque dans les années 1940, comme le précise Jack Tebbutt:

Par exemple, l'homme qui posait des talons, il en avait deux ou trois autres qui travaillaient pour lui pour préparer les talons et assembler les morceaux des parties. Il avait un montant pour préparer et poser les talons. Il engageait ses apprentis, des jeunes, et il payait ces gens-là et ce qui restait était pour lui. ${ }^{24}$

La pratique du «sweating-system» étend, hors des murs de la manufacture, les structures hiérarchiques et l'autorité des patrons. Elle favorise l'intégration d'un sous-prolétariat à la discipline de la production industrielle. Cela prend plusieurs formes et touche des secteurs, parfois secondaires, de la production comme telle.

Une des premières formes de sous-contrat est celle de la fourniture de matériel nécessaire à la fabrication des chaussures, le cuir. Les patrons de la Three Rivers Shoe et, possiblement, ceux de la Smardon avant eux, ont donné des contrats de préparation de cuir fin à des mégissiers de la ville. De même, le matériel pour emballer les chaussures est-il fourni, à compter de 1888 , par P. V. Ayotte. Il s'agit de «boîtes en carton qui sont employées à empaqueter les chaussures». Comme dans bien des cas, les fermmes et les jeunes filles qui travaillent dans ce genre d'entreprise, au service d'une manufacture, sont engagées par l'intermédiaire des petites annonces, répétées à intervalles réguliers jusqu'en $1893^{25}$.

\section{C - La discipline du temps de travail}

Comme on peut le constater, il semble que de 1888 à 1908, la synchronisation des différentes étapes du processus de production doive compter avec les aléas du marché, le rythme de travail des employés et la division de plus en plus poussée des tâches.

La régularisation et la synchronisation du temps de travail ont été obtenues par l'introduction progressive de ce que Thompson nomme

23 Témoignage de Jack Tebbutt, loc. cit.

24 Ibid.

25 Le Trifluvien, 22 décembre 1888, 3; 9 mai 1891, 3; 3 février 1899, 3. 
«la discipline du temps de travail», c'est-à-dire: en arriver à progressivement inculquer aux travailleurs cette conception d'un travail chronométré, mesuré, cette conception d'un travail discipliné.

Les moyens utilisés ont peu varié d'un continent à l'autre: règlements stricts, brimades, amendes, cachots. Les règlements d'usine incarnent tout particulièrement la maîtrise du temps de la part des entrepreneurs. Dans son règlement-type d'une fabrique, Fernand Harvey précise que «les heures de travail, la discipline, la négligence et les départs pouvaient être strictement régis» parce que, écrit-il, «les grandes fabriques avaient senti le besoin de normaliser les différentes conduites de l'ouvrier».

Étant donné les sources que nous avons utilisées, notre étude ne nous a pas permis de recueillir des informations originales à ce sujet. Nous devons référer aux travaux classiques pour une analyse plus détaillée. Nous nous permettons toutefois de souligner que les amendes, en particulier, «illustrent à la fois la volonté d'accumulation du capital, les imperfections de la technique et de l'organisation du travail et le caractère arbitraire des décisions des contremaîtres» ${ }^{26}$. Ces derniers n'hésitent pas à recourir aux menaces et à affronter, souvent seuls, un groupe de travailleurs qui refusent de se plier à la discipline de temps imposée par le patron, comme lors de la grève en 1900.

\section{3 - La formation du temps libre}

On pourrait penser que l'histoire de cette manufacture s'arrête là, qu'elle n'est à tout prendre qu'un cas explicite du processus de formation du temps industriel au Québec à la fin du siècle dernier. Or, l'introduction du temps industriel signifie que des conceptions marchandes du temps ont mené à restructurer autour du temps-pivot du travail, l'ensemble du rythme de la vie en société, à tel point que la vie religieuse et familiale, le sommeil même, en seront profondément affectés.

La mesure du temps de travail a eu comme contrepartie de révéler en quelque sorte, de départager de plus en plus formellement le temps pour l'employeur et le temps personnel. Comme l'écrit Thompson, l'industrialisation «peut être envisagée comme une différenciation technologique violente entre le travail et la vie» ${ }^{27}$, ou plutôt, dirons-nous, entre le temps de travail et les autres sphères de vie.

Temps familial, temps religieux et même temps scolaire en formation, n'occupent pas l'ensemble des temps hors-travail. Il reste toujours une marge relative de temps indéterminé, peu structuré, sinon résiduel. C'est ce temps, à la marge du temps hors-travail, qui a été 13.

26 Fernand Harvey, Révolution industrielle et travailleurs (Montréal, Boréal Express, 1978),

27 E. P. Thompson, The Making of the English Working Class (Harmondsworth, Penguin Books, 1980), 487. 
indistinctement associé, aux débuts de l'industrialisation, au repos, à la récupération physique, au divertissement, à l'éducation. Ce temps libre résiduel, pratiquement sans statut et peu formalisé, s'est vu octroyé des contenus et des significations très variés, d'autant plus que la valorisation du travail condamnait presque automatiquement à des représentations en termes d'oisiveté cette mince frange de temps personnel, affranchie des contraintes du travail, de la famille et de la religion.

C'est bien ce que l'on observe à la manufacture de chaussure de Trois-Rivières, au point qu'on est surpris de constater le rôle joué par l'entreprise dans l'organisation des loisirs ouvriers. Dès son implantation, la Smardon "génère» des activités de temps libre et devient un point de départ d'activités hors travail; cela s'avérera encore plus manifeste avec l'arrivée des frères Tebbutt, étant donnée leur présence soutenue sur la scène sportive et culturelle.

En d'autres termes, la manufacture est le lieu de structuration non seulement du temps de travail, mais aussi d'une frange significative du temps libre. À cet égard, nous avons pu observer deux phases majeures dans l'impact de cette manufacture dans le champ du temps hors travail: une phase sinon d'intense activité, du moins de nouvelles formes de sociabilité, souvent peu organisées, se déplaçant à travers divers lieux publics et servant de prétexte à des démonstrations publiques d'habiletés individuelles ou d'équipes; on a alors l'impression que les nouveaux lieux de travail suscitent diverses activités hors travail, plus ou moins organisées, fragiles, ponctuelles, éphémères. Dans une seconde phase, cependant, les traits marquants sont la structuration des groupes et des activités, la formation de clubs ouvriers et finalement l'avènement d'un «public».

\section{A - Une sociabilité ouvrière informelle: le club sportif Saint-Crispin}

Le club sportif Saint-Crispin de Trois-Rivières, bien qu'il en emprunte le nom, a peu à voir avec l'Ordre des Chevaliers de SaintCrispin, fondé par les artisans de la chaussure ${ }^{28}$. En effet, plutôt que chez les artisans, ce club, qui s'installe à Trois-Rivières en 1889 , recrute ses membres parmi l'élite des employés de la manufacture Smardon, les cordonniers-monteurs et les tailleurs, soit les employés les mieux payés dans l'industrie de la chaussure. Aux différents postes de direction du club, on constate la présence de Canadiens français tandis que les anglophones occupent une place stratégique dans la hiérarchie du club. John-Thomas Tebbutt siège comme trésorier en avril 1890, sans doute le véritable lieu du pouvoir. De même son frère James occupe successivement les fonctions de membre du comité d'organisation en avril 1890, de vice-président et de membre du comité de salle en sep-

28 Don Divance Lescohier, The Knights of Saint-Crispin (1867-1879) (Madison's University of Wisconsin, 1910). 
tembre 1890, mai 1891 et en avril 1892. De son côté, Richard Smardon ne participe pas au club de la compagnie, sinon pour présider l'inauguration du terrain réservé aux membres du club.

En fait, il semble que nous avons affaire à un club social dont les responsables font partie du personnel de direction de la manufacture. Club et manufacture sont si intimement liés que, lors de la première fermeture de la Smardon en 1893 et lors de la fermeture définitive en 1895, les mêmes responsables, les frères Tebbutt, s'impliquent dans un autre club sportif, le TRAAA, dont nous reparlerons.

Les journaux rapportent que l'association a pour but de favoriser le développement physique de ses membres et leur participation à différentes activités sportives ${ }^{29}$. Un des lieux privilégiés par le club se trouve sur le côteau Saint-Louis, au nord de la ville, lequel, à l'instar de quelques autres endroits «ouverts», offre à cette époque un espace propice à des activités à grand déploiement. Déjà en 1829, on y organisait des courses de chevaux. Les membres du club vont officiellement prendre possession, en mai 1890, d'un terrain spécialement aménagé pour leurs tournois, en invitant les Trifluviens à se présenter en nombre pour participer à une journée de joutes sportives qui agrémentent la cérémonie d'inauguration. Ces activités semblent justement plaire aux Trifluviens qui se pressent sur le site chaque fois qu'on leur en donne l'occasion.

Pour les activités d'intérieur, le club dispose de salles où l'on peut pratiquer une variété de jeux de société et, à l'occasion, on invite les amateurs de la ville à se joindre aux membres du club ${ }^{30}$. Lors de la cérémonie d'inauguration du terrain du club, Le Trifluvien précise: «Avant les courses les membres du club Crispin se rendirent à l'hôte! Saint-James où un bouquet magnifique fut présenté au président, M. Ls. Trudel, au nom des dames de Trois-Rivières... ${ }^{31}$ Il est probable que le club avait choisi de s'installer dans cet hôtel.

Dans le même ordre d'idées, même s'il ne s'agit pas du club luimême, on retrouve deux autres lieux possibles de sociabilité pour les employés de la manufacture. Divers documents citent un nommé George Dufresne, hôtelier sur la rue du Platon et un restaurateur, Napoléon Morissette, sur la rue Royale, puis du Platon. Tous deux jugent à propos, en 1899, d'apposer leurs signatures au bas d'une requête des employés, sans doute parce qu'une partie de leur clientèle se recrutait parmi les ouvriers de la Three Rivers Shoe, ex-Smardon ${ }^{32}$. Ces salles,

\footnotetext{
29 Le Trifluvien, 24 août $1889,7$.

Ibid., 10 octobre 1891,3 .

Ibid., 7 juin 1890, 3 .

Archives municipales de Trois-Rivières, Rôles d'évaluation municipale, 1895 et 1900; procès-verbaux, mars 1899.
} 
puisque l'on parle de plusieurs, devaient servir aussi de lieux de lecture et de discussion sur les nouvelles locales, la vie politique, les problèmes du monde ouvrier et autres échanges informels. Ces locaux se trouvent dans le centre-ville du temps, à l'écart de la manufacture et des résidences de la majorité des employés (un décomptage minutieux des listes disponibles d'employés, nous a permis d'établir, par un croisement avec les données des rôles d'évaluation, que la très grande majorité des employés habitaient à proximité de l'usine).

Les activités du club se concentrent dans le sport, dont le «lacrosse»; les tournois de lacrosse demeurent sans doute la manifestation la plus fréquente de la sociabilité organisée autour du temps hors travail. Comme un certain nombre d'employés de la Smardon viennent de Montréal, il est probable qu'ils aient pris l'habitude d'y jouer par l'intermédiaire de clubs montréalais. En fait, durant les années 1870-1880, ce sport connait une certaine popularité parmi la classe ouvrière de Montréal. C'est dans les clubs de lacrosse que les ouvriers pouvaient participer au sport amateur, jusque-là réservé à une certaine élite anglo-saxonne ${ }^{33}$. Il semble en être de même pour le club Crispin. Les parties de lacrosse se jouent normalement entre les employés de la Smardon. Dans un deuxième temps, il arrive que des clubs rivaux de Trois-Rivières et de la région relèvent un défi. Parfois des équipes de l'extérieur se déplacent pour rencontrer les sportifs du club, notamment le club Indépendant de Montréal; de telles parties attirent des spectateurs de partout y compris de Montréal et de Québec. Les supporteurs voyageaient alors par train, les compagnies de chemin de fer accordant des tarifs réduits aux équipes.

Parmi les autres sports pratiqués par les membres du club, il y a aussi les courses et les épreuves de force qui mettent en valeur la forme physique et l'habileté des amateurs. C'est l'occasion pour les citoyens de participer activement. En effet, le club ouvre ses rangs à la population lors de moments particuliers, comme la journée d'inauguration du terrain en juin 1890. De plus, les amateurs du club Crispin s'adonnent à la marche en raquettes durant la saison froide. L'ensemble de ces activités implique un minimum d'organisation l'année durant et donc une certaine stabilité de l'organisation.

Les démonstrations publiques occupent une place importante dans les activités du club. Cette pratique n'est pas particulière aux membres puisque d'autres clubs et des associations ouvrières se manifestent aussi sur la place publique, sous forme de parades et de défilés. Ces marches, au son de la fanfare locale, se font durant les périodes creuses du temps de travail, comme le montre la comparaison des dates des défilés avec le cycle de travail.

${ }^{33}$ Alan Metcalfe, «The Evolution of Organized Physical Recreation in Montreal 18401895», Histoire sociale/Social History, 11,21 (mai 1978): 144-166. 
Le club organise aussi des excursions en bateau sur le fleuve SaintLaurent entre Trois-Rivières et Batiscan. Comme à d'autres occasions, la Société Philharmonique est de la partie. Toutefois, ce genre d'activités, sans doute à cause du coût élevé du billet, ne semble pas attirer beaucoup de participants.

Enfin, il y a les réunions des membres qui se rencontrent à intervalles réguliers. D'août 1889 à avril 1892, l'association tient quatre réunions annuelles où l'on procède à l'élection des divers comités d'organisation et où l'on précise le programme de l'année à venir. Il est fort possible que les membres inscrits au club se réunissaient à d'autres moments plus informels. L'existence de salles réservées aux membres du club permet de constater une sociabilité organisée autour de jeux de société.

L'intérêt de l'étude du club Crispin de Trois-Rivières réside dans l'interaction entre une manufacture et un club sportif composé d'employés de cette même manufacture. Il est hors de question de voir dans le club Crispin un syndicat ouvrier. Il s'agit bel et bien d'un club social de compagnie. Rappelons que la constitution des Chevaliers de Saint-Crispin exclut automatiquement tout travailleur qui accède au poste de contremaître et il en est de même pour les patrons. L'objectif de ces règlements était d'empêcher que les patrons aient accès aux réunions secrètes de l'Ordre. Ainsi, la participation des frères Tebbutt aux réunions et aux joutes sportives du club Crispin démontre qu'il ne s'agit pas d'un syndicat puisque les deux Tebbutt occupaient des postes de direction dans l'entreprise de Richard Smardon.

\section{$B$ - Les loisirs et le sport organisés}

A la fermeture de la Smardon, les frères Tebbutt vont participer à diverses associations, dont le Three Rivers Amateur Athletic Association (TRAAA) et le «Laviolette Skating Rink» ou «Patinoir» Laviolette. La famille Tebbutt a ainsi joué un rôle clef dans la mise sur pied des loisirs modernes à Trois-Rivières. En d'autres termes, les dirigeants d'une des plus importantes usines de Trois-Rivières de la fin du siècle dernier, certes l'une des plus intégrées à l'univers industriel de l'époque, ont aussi été les acteurs principaux de l'organisation d'activités sportives, sociales et culturelles nouvelles, caractéristiques du loisir moderne.

\section{1 - La Three Rivers Amateur Athletic Association (TRAAA)}

Forts de l'expérience acquise dans l'organisation du club Crispin, les frères Tebbutt se retrouvent donc, dès 1895, dans la TRAAA. Ce club sera au cours des années 1893-1899 le lieu de réalisations diverses dans le domaine du sport et du loisir. Et bien qu'il semble avoir été le fait d'une élite locale, anglophone et francophone, son existence dans la société trifluvienne révèle une culture urbaine en formation. 
Les origines du club remontent à juin 1893 alors qu'un groupe de sportifs fondent une association du nom «Amateur». Les membres se réunissent chez des commerçants d'articles de sport. Panneton et Blouin, agents de la maison Brandford, fabricant de bicyclettes, offrent leur magasin pour les réunions. C'est en septembre 1895 que Le Trifluvien présente officiellement l'association sous le nom de la «Three Rivers Amateur Athletic Association». L'année suivante, c'est James Tebbutt, entre autres, qui dépose cette requête devant le conseil municipal:

L'humble requête des soussignés vous expose respectueusement: Qu'ils ont formé une association dans un but de récréation pour l'esprit et de délassement pour le corps; et qu'ils désirent que la dite association soit constituée en corporation civile.

Que le nom de la dite corporation sera l'Association Amateur Athlétique de Trois-Rivières (Three Rivers Amateur Athletic Association).

Que la dite corporation est constituée dans le but de cultiver le sport et les amusements athlétiques $(\ldots)^{34}$

Dès ses débuts, le club tient régulièrement des réunions presque toujours mensuelles. Il s'inscrit aussi dans une perspective canadienne par son adhésion, en février 1897, aux règles édictées par la «Canadian Amateur Athletic Association ${ }^{35}$. Le club possède un terrain, adjacent à l'hôtel de ville et au parc Champlain, en plein coeur de la ville. Il en réserve l'espace, en dehors de ses activités, à des spectacles populaires, acrobates, montreurs d'ours, qui amènent les Trifluviens à fréquenter ses installations.

Le sport, sous toutes ses formes, devient prétexte à des manifestations publiques qui tiennent à la fois de la performance athlétique et du spectacle. On parle volontiers de tours de force de la part d'un dompteur de chevaux ou de l'adresse d'un champion de tir au pigeon d'argile. Le spectacle relève singulièrement de l'attraction foraine. Il est à noter aussi qu'on présente souvent ces activités le mercredi après-midi. Ce moment de la semaine semble être une période de congé pour certains.

Dès octobre 1895, sur le terrain du club, on installe une patinoire en plein air pour y jouer des parties de hockey ou y tenir des mascarades en patin. L'installation couvre une surface de 150 pieds sur 80 . Les membres ont prévu une promenade autour de la patinoire pour permettre à des spectateurs d'assister aux parties de hockey et aux diverses manifestations. Il y a un prix d'entrée avec un tarif réduit pour les familles. La patinoire est ouverte tous les soirs de $7 \mathrm{~h}$ à $10 \mathrm{~h}$. Les organisateurs ont aussi prévu des salles d'attente chauffées, une pour les femmes et une pour les hommes.

34 Archives municipales de Trois-Rivières, procès-verbaux du Conseil, mars 1896.

35 Le Trifluvien, 23 février 1897, 3. 
Même si les membres du club font partie de l'élite, les activités qu'ils organisent sont prétextes à des rassemblements populaires qui prennent une réelle importance dans le milieu trifluvien.

\section{2 - Le «Patinoir» Laviolette (1898-1912)}

La construction d'une patinoire couverte, par les membres du TRAAA et possiblement d'un autre club, le Cercle Palamède ${ }^{36}$, marque une étape dans la commercialisation du loisir sportif sur des bases locales. Le 16 septembre 1898, réunis au magasin des frères Tebbutt, les membres envisagent sérieusement de construire une installation qui répondra mieux aux besoins des sportifs de la ville. Dans les semaines qui suivent, une requête est adressée au conseil municipal. Par une lettre, datée du 14 novembre 1898, les requérants suggèrent aux conseillers de leur accorder une exemption de taxes afin d'avoir toutes les chances possibles de réaliser leur projet. Le même mois, une requête de constitution en corporation civile est déposée auprès du conseil municipal: pour ce qui est des objectifs, elle reprend exactement les termes de la requête du TRAAA précédemment citée ${ }^{37}$. La corporation municipale accepte la requête et reconnaît la compagnie du «Laviolette Skating Rink» en décembre 1898.

Situé dans le quartier Notre-Dame, dans un espace ouvert adjacent à la rue Des Commissaires, il s'agit d'un bâtiment en bois recouvert de tôle et d'une longueur de 180 pieds sur 80 pieds de large (voir illustration). L'ensemble se détache nettement de l'architecture de la ville environnante. On y fait poser plus de 50 lampes électriques, ce qui est sans aucun doute une innovation pour les Trifluviens pour qui l'électricité est encore un luxe.

Les bases de l'organisation de la patinoire sont du type de la compagnie par actions. Les propriétaires de la patinoire offrent en vente, en février 1899, des abonnements ou billets de saison qui donnent le droit de participer aux activités du club. A compter du 30 novembre 1898 , on parlera indistinctement du «Patinoir» Laviolette et du TRAAA. Le groupe va diversifier ses activités tout en conservant les pratiques antérieures de loisirs sportifs et d'exploits athlétiques.

Un clivage entre spectateurs et participants, déjà présent avec le TRAAA, se renforce avec la création du «Patinoir» Laviolette. Qu'il soit question d'une soirée costumée ou d'une partie de hockey, les participants sont membres du club, tandis que les gens extérieurs au groupe peuvent assister, comme spectateurs, aux exploits des élites en payant un prix d'entrée lors de chaque représentation. Cependant, il arrive certains soirs que le public soit admis gratuitement. Et si on s'en

36 Le Trifluvien, 27 août 1901, 3.

37 Archives municipales, procès-verbaux du Conseil, novembre 1898. 


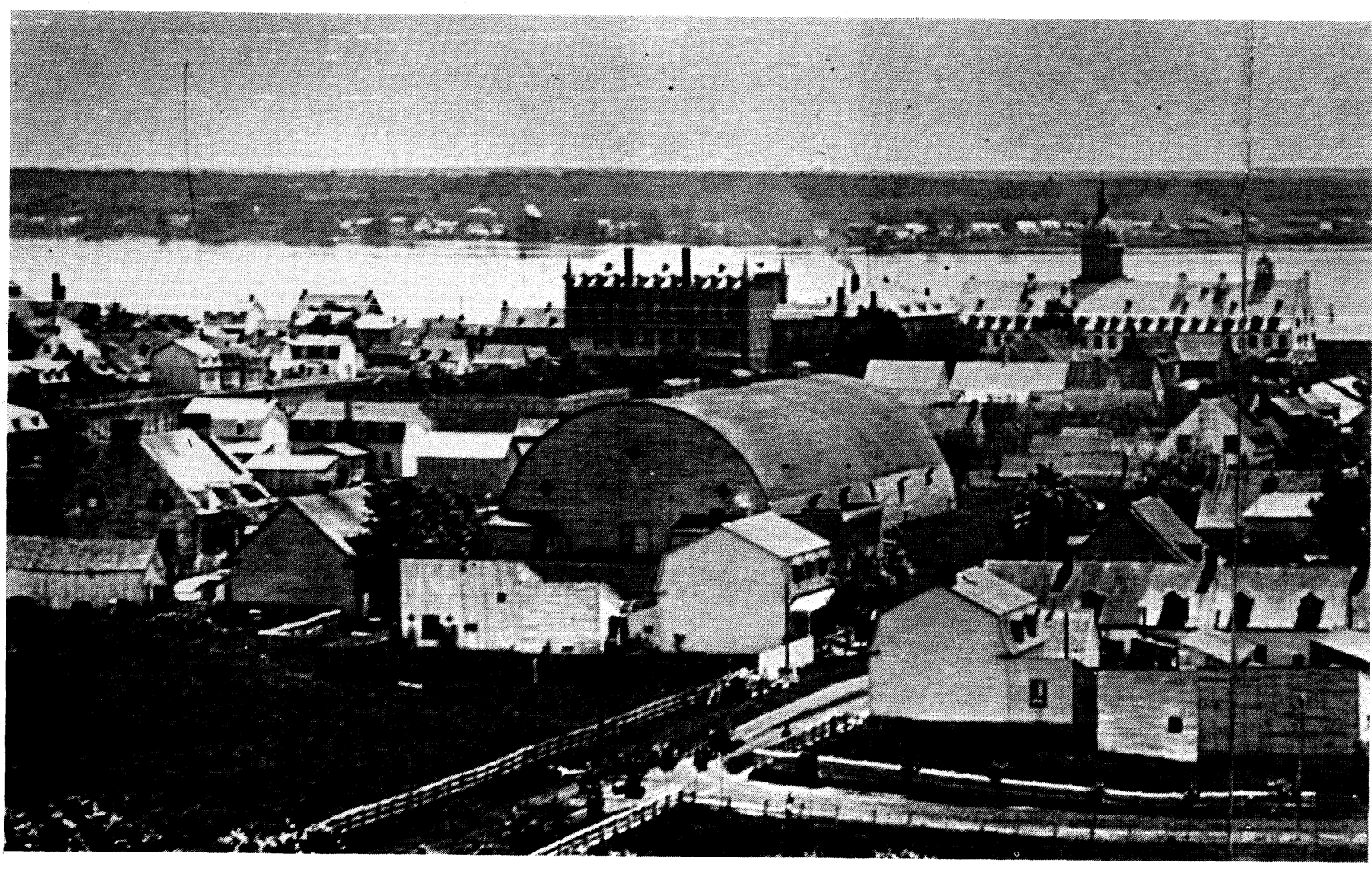

ASTR Vue panoramique de la ville de Trois-Rivières (1900-1903). Cette photographie est tirée d'un montage photographique daté de 1900-1903 et déposé aux archives du Séminaire des Trois-Rivières. L'édifice en bois au toit en demi-cercle est la patinoire couverte dite «Patinoir Laviolette». 
tient aux déclarations des journaux, dès la soirée d'ouverture les gens de la ville affluent pour voir de près la patinoire.

Il y a donc un intérêt réel de la part d'une partie de la communauté trifluvienne, et plus particulièrement des élus municipaux, pour cette nouveauté. A ce titre, il est à remarquer que lors de la cérémonie d'ouverture, et à l'occasion des activités du club, le clergé catholique est toujours absent. La presse locale ne fait jamais mention qu'un dignitaire ecclésiastique ait montré un intérêt quelconque pour cette forme de loisir.

Les directeurs de la patinoire voient loin et tentent de faire de leurs installations une salle polyvalente qui se prêterait à d'autres activités que le sport. Ainsi, en 1901, ils aménagent un théâtre à l'intérieur de la patinoire, ce qui permet d'allonger la période d'utilisation.

Et pourtant, malgré une certaine popularité des spectacles offerts par le «Patinoir» Laviolette, le comité de direction doit songer à fermer ses portes en octobre 1912. Les dépenses encourues pour la construction de la patinoire couverte et l'organisation des divers spectacles obligent le président honoraire, J. Lewis Williams, à entamer les procédures de dissolution de la compagnie le 18 octobre 1912. Un nouveau groupe prend la relève en janvier $1913^{38}$.

\section{3 - Cas typiques d'organisation du loisir (1888-1908)}

Les membres du TRAAA et du «Patinoir» Laviolette s'éparpillent pour ainsi dire dans une foule d'activités sportives. On est encore loin de la spécialisation des clubs comme nous la connaissons aujourd'hui. A la lecture des différents articles de journaux sur les exploits sportifs de ces athlètes, on a l'impression qu'un cercle restreint d'individus est impliqué dans l'organisation de la plupart des formes de sports et de loisirs à la mode. Les exemples qui suivent illustrent les lignes de force qui ont caractérisé la structuration du temps de loisir au tournant du siècle, à Trois-Rivières, principalement par le rôle majeur joué par le TRAAA, dont, ne l'oublions pas, les dirigeants et les ouvriers qualifiés de la manufacture de chaussure ont constitué les acteurs principaux.

\section{Les carnavals}

Un journal fait remarquer en 1884 que: «Le mardi-gras s'est passé absolument sans bruit. L'habitude de faire des mascarades à cette occasion est entièrement disparue parmi nos populations. ${ }^{39}$ La fondation du TRAAA, avec sa première patinoire extérieure adjacente à l'hôtel de ville et face à la cathédrale, va relancer la pratique du carnaval à

$38 \quad$ Le Nouveau Trois-Rivières, 18 octobre 1912, 10; 17 janvier 1913.

39 Le Journal des Trois-Rivières, 28 février 1884, 2. 
Trois-Rivières. Les habitués du patinage répondent à l'invitation du club qui ne lésine pas sur la préparation: «Les organisateurs ont arrangé de grandes courses de patin pour l'occasion. Le programme porte également une grande illumination et un ballon sera lancé. ${ }^{40} \mathrm{Le}$ Trifluvien se plaît volontiers à féliciter les organisateurs: «Le carnaval costumé de vendredi dernier au patinoir TRAAA a été un véritable succès. Plus de 100 costumes sur la glace et près de 800 spectateurs. Recette magnifique, dit-on. ${ }^{41}$ On aura noté le caractère lucratif de la fête puisque le journal se réjouit de la réussite financière de l'événement.

Le TRAAA semble donner trois à quatre carnavals costumés par année. Les membres vont continuer cette pratique avec la fondation du «Laviolette Skating Ring». Les actionnaires du «Patinoir» Laviolette organisent des soirées de mascarade où le bal costumé est à l'honneur. Évidemment, lorsqu'il s'agit de soirée costumée, on parle sans doute d'une élite locale qui prend part d'une manière active à cette forme de loisir. Ici encore, il existe une nette distinction entre les participants et les spectateurs, entre les membres et les non-membres. Toujours d'après la presse locale, ce genre de loisir attire beaucoup de monde tant spectateurs que patineurs. A cela s'ajoute l'Union Musicale, composée de musiciens trifluviens, qui donne un concert tous les lundis soirs durant la saison d'hiver.

En plus des concerts-promenades et des carnavals, les directeurs de la patinoire organisent des spectacles de patin artistique où des champions de calibre international viennent faire la démonstration de leur talent. Mais il n'y a pas que les professionnels qui s'offrent en spectacle. Les amateurs de la ville se prêtent volontiers au jeu du vedettariat: «Les patineurs portant les costumes les plus pittoresques et se livrant à leurs évolutions sous les rayons éclatants de la lumière électrique, ont été admirés par les spectateurs enthousiastes». ${ }^{42}$

Ce type de loisir d'hiver se continue au-delà de la période privilégiée par la présente recherche. Des coups de sonde effectués dans le Nouveau Trois-Rivières, publié à compter de 1908, révèlent qu'on organise encore des carnavals et des mascarades au «patinoir» Laviolette en janvier 1913.

\section{Hockey sur glace}

Après le carnaval et les mascarades, le hockey sur glace semble être la principale activité qui canalise les énergies de l'élite formant le club du TRAAA et du «Patinoir» Laviolette. La fréquence et la régu-

\footnotetext{
$40 \quad$ Le Trifluvien, 14 janvier 1896, 3.

41 Le Trifluvien, 24 janvier 1896, 3.

42 Ibid., 24 janvier 1902, 8.
} 
larité des parties démontrent, une fois de plus, l'ampleur du loisir organisé à Trois-Rivières. Les renseignements fournis par les journaux locaux se résument à des comptes rendus sportifs. Il faut noter cependant que ce sport en est encore à ses débuts à Trois-Rivières à la fin du 19e siècle. Les arbitres ont même des problèmes pour choisir le vainqueur à la fin d'une partie!

Ce qui ressort en premier lieu, c'est l'opportunité pour tous ces amateurs de voyager d'une ville à l'autre et d'établir ainsi des contacts avec les élites d'autres villes. Deuxièmement, et bien qu'il soit difficile de vraiment cerner les caractéristiques de ces joueurs, on peut supposer qu'ils se recrutent parmi la jeunesse de cette élite. Tous ces clubs voyagent par train entre les différents lieux de rencontres. Les joueurs voyagent le soir et le samedi plus particulièrement. Les parties ont lieu vers $8 \mathrm{~h}$. du soir. Il est fréquent de voir un même club jouer deux parties dans un intervalle de 48 heures, ce qui inclut le temps nécessaire pour voyager entre les deux lieux de rencontre. Par exemple, le TRAAA joue à Québec le 6 janvier 1898 et revient à Trois-Rivières le 8 janvier pour rencontrer le West End AAA de Montréal. Même des clubs de l'extérieur du Québec, comme une équipe de Winnipeg, sont parfois inscrits dans ce réseau de relations. Ces échanges sont fréquents. Les clubs voyagent d'une ville à l'autre plusieurs fois dans la même saison. Par exemple du 7 janvier au 8 mars 1898 , on compte cinq clubs étrangers qui rendent visite au TRAAA.

Dès ses débuts, le TRAAA envisage de tirer un profit de ces spectacles sportifs qui attirent le public trifluvien. Dans un premier temps, il semble qu'on veuille tout simplement couvrir les dépenses encourues par un passe-temps coûteux; il s'agit de pourvoir aux dépenses du club social. Mais en parallèle, même s'il est difficile de vraiment distinguer une progression chronologique, on entrevoit la volonté de faire un profit quelconque avec une source de revenus au même titre qu'une entreprise.

En effet, le paiement du prix d'entrée n'est pas exigé uniquement des membres de l'association mais aussi des spectateurs non-membres et, si la joute annoncée promet d'attirer une foule plus nombreuse, on prévoit exiger un supplément pour ceux qui désirent réserver un siège pour la soirée. A l'occasion d'une partie plus importante, qui se tient à l'extérieur de Trois-Rivières, on organise un voyage par train pour permettre aux partisans locaux de suivre leur équipe. C'est que les équipes sportives, en général, et les héros sportifs du monde du hockey, en particulier, suscitent par leurs faits et gestes diverses manifestations de soutien ou de rejet, selon le clan choisi. Dès 1896, la notion d'identification à un club a tout son sens et cet aspect du sport ressort distinctement dans les journaux trifluviens. 
Les moeurs des spectateurs démontrent aussi plus d'une fois la charge émotive que recèle déjà le spectacle sportif à cette époque. Les spectateurs prennent à coeur la réussite du club local, non sans accroc au code d'honneur du bon sportif, au grand désespoir des directeurs du TRAAA puis du «Patinoir» Laviolette. Ainsi Le Trifluvien du 17 janvier 1899 rapporte un article du Daily Telegraph, de Québec, qui critique sévèrement le comportement des spectateurs trifluviens. Ces derniers auraient pris à partie les joueurs de Québec au point de les poursuivre par les rues de la ville jusqu'à la gare de chemin de fer où ils arrivèrent malgré tout sains et saufs, mais de justesse, semble-t-il!

Comme on peut le noter, la violence accompagne parfois ces exploits sportifs; par contre, on encourage les comportements civilisés en félicitant les joueurs qui font preuve de civisme dans le sport:

Superbe partie de hockey au Patinoir Laviolette samedi soir, ... La partie a été jouée élégamment, sans un coup brutal.

On a regretté l'absence de $\mathrm{M}$. Tebbutt, le capitaine de notre équipe, qui n'était pas encore remis des coups reçus à Québec, lors de la partie Laviolette-Crescent. ${ }^{43}$

Il arrive que les membres d'une équipe et leurs supporteurs paradent par les rues de la ville, surtout les plus jeunes de ceux-ci. Et c'est au son des chansons des clubs que les amateurs défilent sous les regards de leurs concitoyens. Le sport est un spectacle qui attire les foules, même lors des matchs en plein air qui se tiennent à la patinoire extérieure du TRAAA. La parodie a aussi sa place. La description qui suit illustre l'idée que le spectacle l'emporte sur le sport:

Grande partie de hockey entre les Pouf et les Ouf sur le terrain de l'Association Athlétique, mercredi soir. Ce ne sont pas des professionnels du hockey... Mais ils ont tout de même déployé une noble ardeur et se sont consciencieusement donné des coups. Tout comme les vieux joueurs quoi $4^{44}$

On aura encore noté l'avènement des professionnels du sport. Comme l'indique la précédente citation, il existe déjà une distinction entre les amateurs et les professionnels. Cela marque de toute évidence une transition vers un modèle plus contemporain du loisir de masse lié au sport. C'est vers 1904 que l'on peut relever l'existence de professionnels du sport à Trois-Rivières. La commercialisation du loisir se fait nettement sentir dans les journaux. Le Trifluvien s'efforce justement, en mars 1904, de justifier la hausse des prix d'entrée, aux parties données au «Patinoir» Laviolette, par la présence d'un entraîneur professionnel ${ }^{45}$.

43 Ibid., 16 janvier 1900, 3.

44 Ibid., 11 mars 1898, 3.

45 Ibid., 4 mars 1904, 4. 
Cette sociabilité autour du sport-loisir fait tache d'huile. Les employés de banque de Trois-Rivières rencontrent les directeurs du «Patinoir» Laviolette, en février 1899, pour une partie de hockey. C'est possiblement la première mention d'un club sportif relié directement au milieu de travail, autre que le club Crispin des employés de la Tebbutt. Dans le cas des employés de banque, il est question de Trifluviens impliqués dans le sport amateur. Ce milieu de travail semble avoir été un lieu propice, à Trois-Rivières comme ailleurs, ainsi que le rapporte Le Trifluvien du 14 mars 1905: «Samedi soir le club de hockey «Trifluvien» a été défait par un club composé des meilleurs joueurs de la Ligue des Banques de Montréal.»

Le «Patinoir» Laviolette ne sert donc pas uniquement à ses propriétaires mais aussi à une multitude de clubs de la ville qui utilisent les installations pour jouer leurs parties. Ainsi, des clubs comme le Cercle Catholique et le Laurier, dont les dénominations indiquent qu'il s'agit de Canadiens français catholiques, pratiquent leur sport au «Patinoir» Laviolette en février 1908 avec un enjeu monétaire.

Finalement, un dernier détail vient renforcer l'impression d'une intégration de la notion de temps de loisir. Vers 1905, un calendrier des joutes et une liste des clubs en compétition sont publiés par Le Trifluvien $^{46}$. Il est donc certain que dès cette époque les citadins de Trois-Rivières sont familiarisés avec une certaine organisation du temps de loisir sur des bases régulières.

\section{CONCLUSION}

A la suite de tractations coutumières entre les membres du Conseil municipal et des hommes d'affaires, une entreprise de chaussures, sans doute semblable à beaucoup d'autres, est venue s'implanter à TroisRivières à la fin du siècle dernier. Son histoire révèle un temps industriel en formation, une pénétration progressive dans l'univers du travail discipliné et segmenté; la population ouvrière de Trois-Rivières faisait peu à peu l'expérience d'une nouvelle culture du travail et de l'entreprise, de nouvelles hiérarchies socio-économiques, ainsi qu'une appropriation différente de l'espace urbain; et ce, à l'instar de ce qui se passait sans doute dans la plupart des agglomérations urbaines de l'époque.

Mais l'histoire ne s'arrête pas là. Ce qui se produit, c'est la restructuration de l'ensemble des temps sociaux, de par l'émergence du travail comme temps-pivot des sociétés industrielles. Si l'on a relativement bien étudié la genèse et la formation du temps industriel, on a moins souvent pris en considération le processus qui s'étend, pour ainsi dire, aux autres temps sociaux.

\footnotetext{
${ }^{46}$ Ibid., 10 janvier $1905,5$.
} 
Dans le cas du temps libre, en particulier, nous avons voulu décrire l'impact remarquable, sinon décisif, des dirigeants d'une entreprise de chaussures et des ouvriers qualifiés, sur la structuration du temps hors travail. S'inscrivant avec une très grande aisance dans les cercles de la bourgoisie locale, multipliant les initiatives, ils ont été les principaux auteurs de nouvelles activités, de nouvelles formes de sociabilité, et ils ont servi de relais, à Trois-Rivières, au mouvement alors observable de structuration et d'institutionnalisation de temps libre. Incidemment, nos observations et nos conclusions rejoignent ce qui a été souligné dans le cas de Montréal, pour une période antérieure ${ }^{47}$.

C'est pourquoi, on peut en conclure que changements économiques et changements culturels vont de pair. La structuration du temps de travail a eu son corrolaire: la structuration du temps de loisir; la discipline du temps de travail a contribué à démarquer les autres temps sociaux.

\footnotetext{
47 Alan Metcalfe, «Le sport au Canada français au 19e siècle: le cas de Montréal (18001914)», Loisir et société/Society and Leisure, 6,1 (1983): 105-120. Nous nous permettons également de référer à notre ouvrage qui a développé cette problématique: Gilles Pronovost, Temps, culture et société (Presses de l'Université du Québec, 1983), particulièrement les chapitres 1 et 4.
} 